\title{
Le polymorphisme d'insertion/délétion du gène de l'enzyme de conversion de l'angiotensine I semble être un facteur de risque d'infarctus du myocarde
}

Les cardiopathies ischémiques sont une des principales causes de morbidité et de mortalité précoce dans les sociétés occidentales. Le nombre annuel d'infarctus du myocarde en France est d'environ 110000 , dont 38000 cas chez l'homme et $8500 \mathrm{chez}$ la femme avant 65 ans. Un homme de 45 ans a $20 \%$ de chance de faire un infarctus du myocarde avant 75 ans; chez la femme, ce pourcentage est de $10 \%$. La France, pourtant, est loin d'être le pays le plus touché par ce fléau. La fréquence de l'infarctus avant 65 ans est en effet 3 à 4 fois plus importante dans certains pays du nord de l'Europe comme la Finlande, l'Écosse ou l'Irlande du Nord qu'en France. Aux États-Unis d'Amérique, le nombre annuel d'infarctus du myocarde dépasse encore largement le million, malgré la remarquable réduction de fréquence enregistrée au cours des vingt dernières années. Ces quelques chiffres illustrent la dimension du problème de santé publique posé par cette maladie dont les mécanismes sont relativement bien connus, mais dont les déterminants génétiques et environnementaux sont loin d'être totalement élucidés.

La nécrose localisée du muscle cardiaque, qui caractérise l'infarctus du myocarde, est consécutive à l'obstruction complète d'une des artères nourricières du cœur (coronaires) survenant brutalement sur une lésion d'athérosclérose. L'athérosclérose est une maladie chronique de l'artère qui évolue sur des dizaines d'années et qui est favorisée par certains troubles du métabolisme des lipoprotéines dont l'étiologie est à la fois génétique et nutritionnelle. Les artères coronaires d'un individu adulte peuvent porter plusieurs plaques d'athérosclérose, plus ou moins étendues et obstruant plus ou moins la lumière artérielle. Un facteur majeur de complication de la plaque est sa fissuration et sa rupture, la thrombose locale qui en résulte contribue à la progression de la lésion d'athérosclérose et joue un rôle essentiel dans la pathogenèse de l'angor instable, de l'infarctus du myocarde et de la mort subite. Des travaux angiographiques ont par ailleurs montré que des phénomènes de vaso-constriction aigus ou subaigus accompagnent les ruptures de plaques d'athérosclérose et, associés à la thrombose, contribuent à la survenue des syndromes coronariens aigus. Un vasospasme chronique, même modeste, pourrait aussi, en modifiant les caractéristiques du flux sanguin coronaire et secondairement la structure de la paroi vasculaire, favoriser l'apparition de phénomènes ischémiques sur des lésions d'athérosclérose en moyenne moins évoluées que si l'artère ne présentait pas de spasme.

L'importance des anomalies du métabolisme des lipoprotéines dans le développement de l'athérosclérose est bien établi. Il est très probable que l'épidémie de cardiopathies ischémiques que connaissent les pays industrialisés occidentaux a pour cause essentielle l'apport considérable de graisses saturées qui caractérise le mode alimentaire

de ces pays. Pourtant, la mise en évidence des facteurs génétiques qui prédisposent à l'athérosclérose ou favorisent ses complications constitue un objectif de recherche important pour mieux comprendre les mécanismes physiopathologiques de la maladie et pour moduler sa prévention et son traitement. De nombreuses équipes sont impliquées dans la recherche des déterminants génétiques de l'infarctus du myocarde et de ses facteurs de risque. Leurs efforts commencent à porter leurs fruits dans le domaine des dyslipidémies, de l'hypertension artérielle et du diabète où de nombreux gènes candidats sont explorés. Depuis quelques années, nous étudions le gène de l'enzyme de conversion de l'angiotensine (ACE), qui nous semble être un excellent gène candidat des cardiopathies ischémiques.

L'ACE intervient dans la production d'angiotensine II, peptide fortement vaso-presseur stimulant la sécrétion d'aldostérone, et dans la dégradation de la bradykinine, peptide vasodilatateur impliqué dans les réactions inflammatoires. L'ACE est ancrée à la membrane de nombreux types cellulaires, en particulier celle des cellules endothéliales vasculaires, et les résultats de certains travaux suggèrent que son effet sur les peptides vaso-presseurs ainsi que l'activité de ces derniers seraient en partie locaux.

L'ACE est également retrouvée sous forme circulante. Le taux circulant de l'enzyme et son activité sont très stables chez un même individu mais 
Tableau 1

DISTRIBUTION DES GÉNOTYPES DE L'ACE CHEZ LES PATIENTS AYANT EU UN INFARCTUS DU MYOCARDE ET CHEZ LES TÉMOINS DE L'ÉTUDE ECTIM SUIVANT QUE LES SUJETS PEUVENT ÊTRE CONSIDÉRÉS A HAUT OU A BAS RISQUE

\begin{tabular}{|c|c|c|c|c|c|c|c|c|c|c|}
\hline & \multicolumn{2}{|c|}{ Belfast } & \multicolumn{2}{|c|}{ Lille } & \multicolumn{2}{|c|}{ Strasbourg } & \multicolumn{2}{|c|}{ Toulouse } & \multicolumn{2}{|c|}{ Tous } \\
\hline & malades & témoins & malades & témoins & malades & témoins & malades & témoins & malades & témoins \\
\hline Génotype ACE & \multicolumn{10}{|c|}{ Groupe considéré à bas risque (1) } \\
\hline $\begin{array}{l}\text { DD } \\
\text { ID + II }\end{array}$ & $\begin{array}{l}13 \\
15\end{array}$ & $\begin{array}{l}12 \\
40\end{array}$ & $\begin{array}{l}5 \\
1\end{array}$ & $\begin{array}{l}11 \\
28\end{array}$ & $\begin{array}{l}10 \\
14\end{array}$ & $\begin{array}{r}7 \\
28\end{array}$ & $\begin{array}{l}10 \\
11\end{array}$ & $\begin{array}{l}16 \\
47\end{array}$ & $\begin{array}{l}38 \\
41\end{array}$ & $\begin{array}{r}45 \\
143\end{array}$ \\
\hline \multirow[t]{2}{*}{ Risque relatif (3) } & \multicolumn{2}{|c|}{$2,9(1,0-8,7)$} & \multicolumn{2}{|c|}{$12,7^{(4)}$} & \multicolumn{2}{|c|}{$2,9(0,8-10,7)$} & \multicolumn{2}{|c|}{$2,7(0,8-8,4)$} & \multicolumn{2}{|c|}{$3,2(1,7-5,9)^{(5)}$} \\
\hline & \multicolumn{10}{|c|}{ Groupe considéré à haut risque (2) } \\
\hline $\begin{array}{l}D D \\
I D+\|\end{array}$ & $\begin{array}{r}38 \\
135\end{array}$ & $\begin{array}{l}31 \\
97\end{array}$ & $\begin{array}{l}22 \\
30\end{array}$ & $\begin{array}{l}32 \\
77\end{array}$ & $\begin{array}{r}57 \\
123\end{array}$ & $\begin{array}{r}48 \\
111\end{array}$ & $\begin{array}{l}42 \\
84\end{array}$ & $\begin{array}{r}43 \\
105\end{array}$ & $\begin{array}{l}159 \\
372\end{array}$ & $\begin{array}{l}154 \\
390\end{array}$ \\
\hline Risque relatif & \multicolumn{2}{|c|}{$0,9(0,5-1,6)$} & \multicolumn{2}{|c|}{$1,8(0,8-3,7)$} & \multicolumn{2}{|c|}{$1,1(0,7-1,7)$} & 1,210 & $7-2,1)$ & \multicolumn{2}{|c|}{$1,1 \quad(0,9-1,5)$} \\
\hline
\end{tabular}

(1) Individus sans surpoids (indice de quetelet $<26 \mathrm{~kg} / \mathrm{m}^{2}$ ), taux plasmatique d'ApoB $<125 \mathrm{mg} / \mathrm{dl}$, et non traités avec des médicaments hypolipidémiants.

(2) Individus qui ne sont pas dans le groupe à bas risque.

(3) Intervalles de confiance à $95 \%$ entre parenthèses.

(4) Test de Fisher exact : $p=0,03$.

(5) Risque relatif ajusté sur la population.

Analyse statistique: Génotype $D D$ plus fréquent chez les malades que chez les témoins : $p<0,0001$. Association entre le génotype DD et l'infarctus plus forte dans le groupe à haut risque que dans le groupe à bas risque : $p<0,001$.

varient fortement entre individus. Nous avons pu montrer il y a quelques années, en analysant la ressemblance familiale du taux d'ACE, qu'il était largement génétiquement déterminé et principalement sous le contrôle d'un seul gène [1]. En effet, $50 \%$ de la variabilité interindividuelle de l'ACE plasmatique pouvait être expliquée par un effet génétique majeur. Bien évidemment une telle analyse de ségrégation ne permettait pas de dire quel gène était en cause dans la variation observée. Par ailleurs, une étude effectuée par François Alhenc-Gelas et al. (Inserm U. 367, Paris, France) dans un groupe de 434 hommes en bonne santé ne montrait aucune influence de facteurs environnementaux ou hormonaux sur le taux circulant d'ACE [2]. Le clonage de l'ADNc de l'ACE à l'Inserm U. 36 (Paris, France) [3] a permis d'identifier un polymorphisme d'insertion/délétion (ACE/ID) du gène, dont les allèles avaient une fréquence à peu près identique et qui s'est avéré fortement lié au taux circulant d'ACE [4]. Dans une étude d'association portant sur 80 sujets, le taux des homozygotes pour l'insertion (II), des hétérozygotes (ID) et des homozygotes vement de 299, 393 et $494 \mu \mathrm{g} / \mathrm{l}$, la différence entre les trois génotypes étant statistiquement très significative. $\mathrm{Ce}$ polymorphisme du gène de l'ACE avait donc un effet codominant fort sur le taux circulant de l'enzyme. Il était utile à ce stade de savoir si l'effet génétique majeur démontré dans la précédente étude et l'effet du polymorphisme ACE/ID identifiaient le même variant génétique. Une nouvelle étude familiale fut donc réalisée, toujours en collaboration avec les chercheurs du Centre de médecine préventive de Vandœuvre-lès-Nancy, en prenant soin cette fois de conserver l'ADN des individus étudiés [5]. Une analyse statistique couplant ségrégation et liaison (réalisée par Laurence Tiret, Inserm U. 258, Paris, France) permit de montrer que le polymorphisme ID n'expliquait pas entièrement la variabilité du taux circulant d'ACE mise en évidence par l'analyse de ségrégation. Le polymorphisme n'étant probablement qu'un marqueur en déséquilibre de liaison avec un variant fonctionnel inconnu situé dans ou à proximité du gène de l'ACE. Ce marqueur paraissait néanmoins excellent, puisque les auteurs estimaient que la sensibilité de l'allèle D pour identifier l'allèle fonc- tionnel associé à l'augmentation du taux d'ACE était de $100 \%$ et sa spécificité, de $77 \%$.

Étant donnés l'implication de la vasoconstriction dans l'infarctus du myocarde, l'effet vaso-constricteur potentiel des taux élevés d'ACE et l'importance de l'association entre le polymorphisme ACE/ID et le taux d'ACE circulante, il était logique d'envisager que le polymorphisme pût être associé à une prédisposition à l'insuffisance coronarienne. Nous avons donc étudié la fréquence des génotypes du polymorphisme ACE/ID chez des patients atteints d'infarctus du myocarde $(n=610)$ et chez des témoins $(n=733)$ participant à l'étude ECTIM*. Les résultats de ce travail viennent d'être publiés [6]. Ils montrent que le génotype DD est plus fréquemment observé chez les malades que chez les témoins $(p<0,007)$, en particulier chez les sujets à bas risque, non hyperlipidémiques et de corpulence basse (Tableau I). Chez ces individus, en effet, le risque relatif d'infarctus du myocarde associé au génotype DD est d'environ 3 dans les quatre populations étudiées. Cette association très significative $(p<0,0001)$ est indépendante des facteurs de risque classiques, en 
particulier de la pression artérielle, dont le niveau moyen ne diffère pas entre les trois génotypes. L'impact populationnel du polymorphisme peut être estimé à partir du risque relatif et de la fréquence du génotype DD dans la population (environ $27 \%$ ). Il est de $8 \%$ pour l'ensemble des infarctus et de $30 \%$ chez les sujets à bas risque. $\mathrm{Ce}$ risque attribuable représente le pourcentage des cas d'infarctus qui seraient prévenus si l'on supprimait l'effet délétère du génotype ACE/DD dans la population. Bien évidemment, ces résultats devront être vérifiés dans d'autres études et dans d'autres populations avant de pouvoir être considérés comme un fait épidémiologique établi.

L'augmentation du risque d'infarctus chez les sujets ACE/DD pourrait être la conséquence d'une vaso-constriction chronique coronaire ou d'une prédisposition au spasme liée à l'action de l'ACE sur les peptides vaso-actifs qu'il métabolise. En présence de vasoconstriction coronaire, un infarctus du myocarde pourrait survenir sur une lésion d'athérosclérose de taille moins importante qu'en absence de vasoconstriction. Bien que cette hypothèse soit séduisante, il faut reconnaître que d'autres mécanismes pourraient intervenir. En effet, l'angiotensine II peut promouvoir la croissance des cellules musculaires lisses myocardiaques et vasculaires, et induire une hyperplasie

\footnotetext{
* ECTIM ("Étude cas-témoin de l'infarctus du myocarde ") a pour objectif d'identifier des facteurs génétiques impliqués dans l'infarctus du myocarde et d'étudier leurs interactions. Environ 650 malades et 750 témoins ont èlé recrutés dans quatre régions couvertes par des registres de cardiopathies ischémiques participant au projet MONICA de l'OMS en France, à Lille (Drs G. Luc et P. Amouyal), a Strasbourg (Dr D. Arveiler et Pr. Schaffer), et a Toulouse (Drs J.P. Combou et J.B. Ruidavets) et en Irlande du Nord, a Belfast (Pr. A. Evans). Dass ce denier pays, la fróquence de l'infarctus du myocarde est 3 a 4 fois supérieure à celle de la France. Un nombre important d'investigations biologiques ont été réalisées a partir de la banque de plasma mise en place pour cette étude, en particulier au laboratoire du SERLLA (Inserm U. 325, Université Pasteur de Lille) (Dr J.M. Baral et Pr. J.C. Fruchart). D'autre part, six laboratoires partiapent aux explorations génétiques. La banque d'ADN est stockée au service commun $n^{\circ} 7$ de l'Inserm qui a également à sa charge l'exploration des gènes des apolipoprotéines $A 1, C 3, A 4, B, d u$ fibrinogène, de l'ACE et de l'azriolensinogène. L'étude ECTIM se prolonge actuellement par une étude de familles à haut risque à Belfast et par une étude de cohorte portant sur 10000 individus dans les quatre populations coordonnée par le Dr J.L. Richard de l'Inserm U. 258 (Paris, France).
}

néo-intimale en présence d'une artère lésée; la bradykinine quant à elle, outre son activité vaso-dilatatrice, a également une activité antiproliférative. Par ailleurs, rien ne prouve que les variations interindividuelles importantes de l'ACE circulante ou même cellulaire affectent différentiellement la dégradation de la bradykinine ou la conversion de l'angiotensine I en angiotensine II. L'ACE est habituellement considérée comme non limitante dans la cascade métabolique du système rénine-angiotensine. Cette notion importante dans la régulation à court terme du système n'a peut-être aucune pertinence en présence d'un effet chronique comme celui que nous postulons. En outre, l'enzyme pourrait être limitante dans certains territoires artériels.

Pourquoi le polymorphisme de l'ACE n'affecte-t-il pas la pression artérielle ? Logiquement, une vaso-constriction chronique du territoire artériel devrait induire une augmentation des résistances périphériques et rénales, et induire une élévation du niveau tensionnel. On sait également que l'inhibition de l'ACE est l'un des traitements les plus efficaces de l'hypertension artérielle. Certains territoires artériels (dont le système coronaire) seraient-ils plus sensibles à la variation du taux d'ACE ? Un stimulus local, tels la présence ou la complication d'une lésion d'athérosclérose, est-il nécessaire pour qu'un spasme survienne? Le rein ne seraitil pas affecté par la vaso-constriction et aurait-il un rôle compensateur ?...

Si nos résultats sont confirmés, il restera à identifier le variant fonctionnel porté par l'allèle ACE/D. Il faudra aussi quantifier avec précision l'importance de ce nouveau facteur de risque, en conjonction avec les autres facteurs prédisposants à l'infarctus du myocarde et étudier les intérêts respectifs du variant génétique et du taux circulant d'ACE dans l'évaluation du risque. Pour cela, il sera nécessaire de suivre une cohorte d'individus non malades pendant de nombreuses années (étude prospective), une étude cas-témoin telle ECTIM n'étant pas bien adaptée à ce type d'évaluation. Alors peut-être serat-il possible d'envisager une prévention sur un sous-ensemble des individus porteurs du variant, éventuellement par prescription d'un inhibiteur de l'ACE.
Plus rapidement, on devrait savoir si, en post-infarctus, la prévention de la récidive ou de l'insuffisance cardiaque par les inhibiteurs de l'ACE est plus efficace chez les sujets porteurs du génotype ACE/DD que chez les autres. Dans ce contexte, il est intéressant de noter que les résultats récents d'un essai de prévention secondaire montrent que les inhibiteurs de l'ACE ont un effet préventif sur la récidive d'infarctus du myocarde [7]

\section{François Cambien}

Inserm SC 7 et U. 258, 17, nue du Fer à Moulin, 75005 Paris, France

Florent Soubrier

Inserm U. 36, 3, nue d'Ulm, 75005 Paris, France.

\section{RÉFÉRENCES}

1. Cambien F, Alhenc-Gelas F, Herbeth B et al. Familial ressemblance of plasma angiotensin-converting enzyme level: the Nancy study. Am J Hum Genet $1988 ; 43$ : 774-80.

2. Alhenc-Gelas F, Richard J, Courbon D, Warnet JM, Corvol P. Distribution of plasma angiotensin I-converting enzyme levels in healthy men : relationship to environmental and hormonal parameters. J Lab Clin Med $1991 ; 117$ : 33-9.

3. Soubrier F, Alhenc-Gelas F, Hubert C, et al. Two putative active centers in human angiotensin I-converting enzyme revealed by molecular cloning. Proc Natl Acad Sci USA 1988 ; 85 : 9386-90.

4. Rigat B, Hubert C, Alhenc-Gelas F, Cambien F, Corvol P, Soubrier F. An insertion/deletion polymorphism in the angiotensin I-converting enzyme gene accounting for half the variance of serum enzyme levels. $J$ Clin Invest 1990 ; 86 : 1343-6.

5. Tiret L, Rigat B, Visvikis S, et al. Evidence from combined segregation an linkage analysis, that a variant of the angiotensin Iconverting enzyme (ACE) gene controls plasma ACE. Am J Hum Genet 1992 ; 51 : 197-205.

6. Cambien F, Poirier O, Lecerf L, et al. Deletion polymorphism at the angiotensinconverting enzyme gene is a potent risk factor for myocardial infarction. Nature 1992 ; 359 : $641-4$

7. Pfeffer MA, Braunwald E, Moyé IA, et al. Effect of captopril on mortality and morbidity in patients with left ventricular dysfunction after myocardial infarction. $N$ Engl $J \mathrm{Med}$ 1992 ; 327 : 669-77. 